\title{
COMISIÓN POR SALDO Y NIC 18 ¿BENEFICIA A LOS AFILIADOS O A LAS AFP?
}

\author{
COMMISSION FOR BALANCE AND NIC 18 \\ ¿THIS BENEFITS TO MEMBERS OR TO THE AFP? \\ José Salvador Romero Cerna * \\ Docente Auxiliar de la Facultad de Ciencias Contables \\ Universidad Nacional Mayor de San Marcos-UNMSM / Lima-Perú
}

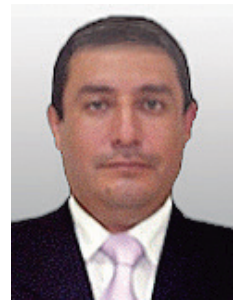

[Recepción: Marzo de 2014/ Conformidad: Mayo 2014]

\section{RESUMEN}

La reciente reforma del Sistema Privado de Pensiones incorpora, entre otros cambios, la comisión sobre el saldo del fondo administrado (comisión por saldo) en reemplazo de la comisión sobre la remuneración (comisión por flujo) que cobran las Administradoras de Fondos de Pensiones (AFP) a los afiliados, y la implementación gradual de la NIC 18 Ingresos de Actividades Ordinarias.

El artículo pretende determinar si los cambios incorporados benefician a los afiliados o a las AFP. Para ello se revisan las diferencias entre las propuestas de reforma del gobierno y la reforma finalmente aprobada, así como, su incidencia en los afiliados y las AFP.

\section{Palabras Clave:}

Reforma del sistema privado de pensiones; conflictos de interés; mercado cautivo; competencia; transparencia; NIC 18.

\begin{abstract}
The recent reform of the private pension system incorporates, among other changes the commission on the balance of the administered funds (commission for balance) in replacement of the Commission on the remuneration (commission flow) charged by the Pension Fund Administrators (AFP) affiliates, and the gradual implementing of NIC 18 Revenue.

The article aims to determine whether changes incorporated benefit to affiliates or the AFP. To do this, the differences between the proposed government reform and reform finally approved, as well as its impact on the members and the AFP are reviewed.
\end{abstract}

\section{Keywords:}

Reform of the private pension system, conflicts of interest, captive market, competition, transparency, NIC 18.

\footnotetext{
* Magíster en Economía con mención en Finanzas - UNMSM. Email: jromeroc5@unmsm.edu.pe
} 


\section{INTRODUCCIÓN}

Han transcurrido más de 20 años desde la creación del Sistema Privado de Administración de Fondos de Pensiones (SPP), constituido por las Administradoras Privadas de Fondos de Pensiones (AFP), mediante el Decreto Ley No 25897 publicado el 06 de diciembre de 1992 durante el denominado Gobierno de Emergencia y Reconstrucción Nacional presidido por Alberto Fujimori.

Mediante Ley $\mathrm{N}^{\circ} 27328$ publicada el 24 de julio de 2000 durante el segundo gobierno de Fujimori se incorpora a las AFP bajo el control y supervisión de la hoy Superintendencia de Banca, Seguros y AFP (SBS) ${ }^{1}$.

Mediante el Decreto Supremo N ${ }^{\circ}$ 054-97-EF, publicado el 14 de mayo de 1997, se aprobó el Texto Único Ordenado de la Ley del Sistema Privado de Administración de Fondos de Pensiones (SPP).

\section{PROPUESTA ELECTORAL DEL GOBIERNO}

La Comisión del Plan de Gobierno 2011-2016 de la alianza electoral Gana Perú ${ }^{2}$ denominado "La Gran Transformación” en el numeral 4.4.3 referida a las Propuestas de Politica sobre la protección y seguridad social y previsional, señala respecto al SPP:

- Libre afiliación y libre desafiliación o traslado en los sistemas de pensiones.

- Promoveremos la participación de los representantes de los afiliados en los directorios de las empresas administradoras de fondos de pensiones, los cuales serán elegidos por sus respectivas asociaciones.

Adicionalmente, en el numeral 7.2.3 Trabajo Decente y Pensiones Dignas como acciones inmediatas, entre otros, propone la implementación de un régimen de pensiones sustentado en dos sistemas:

Un primer sistema público que contiene dos componentes: i) el contributivo-obligatorio (base del sistema) para todos los trabajadores activos, tanto asalariados como independientes cuya aportación estará en función de sus salarios o ingresos; $y$ ii) el no contributivo, que ofrece una pensión básica para todos los adultos mayores de 65 años, financiada básicamente por impuestos y por una porción de los fondos aportados por los trabajadores.

Un segundo sistema privado que sirva de complemento al primero. Este pilar es voluntario y está basado en cuentas individuales para trabajadores asalariados e independientes. Adicionalmente, a la pensión del pilar público cada contribuyente recibe una pensión en función de lo aportado y el rendimiento del fondo escogido.

La propuesta del plan de gobierno era que el sistema público sea el principal régimen de pensiones (obligatorio) y el sistema privado sea complementario (voluntario), y proponía la libre afiliación y desafiliación o traslado en ambos sistemas.

Luego de la primera vuelta electoral la alianza Gana Perú presentó el documento Lineamientos Centrales de Política Económica y Social para un Gobierno de Concertación Nacional, la denominada Hoja de Ruta, en reemplazo del Plan de Gobierno inicial, donde se precisa que el programa PENSIÓN 65 será focalizado y financiado con recursos del tesoro público, $y$ que no se tocarán los fondos de los afiliados de las AFP. Con ello, la alianza buscaba desvirtuar la información en los medios de comunicación respecto a la intangibilidad de los fondos de pensiones ${ }^{3}$.

Una vez en el gobierno, la propuesta más importante la hicieron los congresistas oficialistas Javier Diez Canseco Cisneros ${ }^{4}$ y Jaime Delgado Zegarra. El proyecto de Ley 474/2011CR denominado Ley de fortalecimiento y transparencia del sistema privado de pensiones para beneficio de los usuarios y afiliados, señala como objetivos, entre otros, (i) la implantación del registro de los ingresos de las administradoras (AFP) por concepto de comisiones según las Normas Internacionales de Contabilidad (NIC), y (ii) la promoción de la libre competencia en la provisión del servicio de administración de fondos de pensiones abriendo el marco

1 Mediante Ley $\mathrm{N}^{\circ} 28484$ publicada el 05 de abril de 2005, se modificó constitucionalmente la denominación de la "Superintendencia de Banca y Seguros" por la de "Superintendencia de Banca, Seguros y Administradoras Privadas de Fondos de Pensiones".

2 Alianza conformada por el Partido Nacionalista, Partido Socialista y otras organizaciones políticas de izquierda.

$3 \mathrm{Al}$ respecto el diario El Comercio publica en su portal "En el tema de la AFP, Humala no aclara si tocará los fondos aportados por los ciudadanos"

(19.04.2011), y el Instituto Peruano de Economía (IPE) señala que la famosa Pensión 65 también ha mutado (Comentario Diario del 17.05.2011).

4 El congresista Diez Canseco, fallecido el 04.05.2013, postuló como invitado en la lista parlamentaria de Gana Perú, y posteriormente, renunció a la bancada oficialista el 04.06.2012. 
regulatorio para que las instituciones financieras puedan ofrecer dichos productos financieros.

Si bien, el proyecto de ley no proponía la libre desafiliación y que el sistema privado sea complementario al sistema público, proponía la creación como modalidad de pensión a otros productos financieros con fin previsional brindados por empresas del sistema financiero, así como la participación de estas como administradores de productos financieros con fin previsional, y la implantación de la NIC 18 Ingresos de Actividades Ordinarias que permita a los afiliados el endoso a otra AFP o institución del importe del ingreso diferido correspondiente a su cuenta individual de capitalización (CIC) 5 .

\section{REFORMA DEL SPP EJECUTADA POR EL GOBIERNO}

Mediante Ley N² 29903 (Ley de Reforma del SPP) publicada el 19.07.2012 establece, entre otros, la licitación del servicio de administración de cuentas individuales de capitalización para los trabajadores que se incorporen al SPP, la afiliación del trabajador independiente al SPP y la aplicación de solo la comisión sobre el saldo del Fondo de Pensiones, en un plazo que establecerá la SBS 6 .

Respecto a la comisión cobrada por las AFP, la reforma consiste en la aplicación de la comisión por saldo y la implementación gradual de la NIC 18 , y no la promoción de una mayor competencia con el ingreso de empresas del sistema financiero en la administración de productos financieros con fin provisional y la implantación (no gradual) de la NIC 18, según la propuesta de los congresistas Diez Canseco y Delgado.

\section{NIC 18 Y NORMAS CONTABLES APLICABLES A LAS AFP}

La Norma Internacional de Contabilidad 18 Ingresos de Actividades Ordinarias trata sobre los ingresos que surgen en el curso de las actividades ordinarias de la entidad y adoptan una gran variedad de nombres, tales como ventas, comisiones, intereses, dividendos y regalías.

Los aspectos más significativos del reconocimiento de los ingresos por prestación de servicios son los siguientes:

Los servicios pueden prestarse en el transcurso de un único periodo o a lo largo de varios periodos contables (párrafo 4).

Cuando el resultado de una transacción, que suponga la prestación de servicios, pueda ser estimado con fiabilidad, los ingresos de actividades ordinarias asociados con la operación deben reconocerse, considerando el grado de terminación de la prestación final del periodo sobre el que se informa (párrafo 20).

Cuando el resultado de una transacción, que implique la prestación de servicios, no pueda ser estimado de forma fiable, los ingresos de actividades ordinarias correspondientes deben ser reconocidos como tales sólo en la cuantía de los gastos reconocidos que se consideren recuperables (párrafo 26).

Hasta antes de la Reforma del SPP los ingresos por comisiones de las AFP por la administración del fondo de pensiones, cobradas en base a un porcentaje de la remuneración asegurable del afiliado (comisión por flujo), se reconocían como ingresos devengados luego de haber conciliado y acreditado los aportes de los afiliados en sus cuentas individuales de capitalización, de acuerdo con las normas contables establecidas por la SBS ${ }^{7}$.

\footnotetext{
5 El Instituto Peruano de Economía (IPE) respecto a la aplicación de la NIC 18 señala que es importante que se considere un período de transición para el cambio de sistema, ya que pretender hacerlo de inmediato generaría serios e innecesarios problemas (Comentario Diario del 13.02.2012).

6 El artículo 4 de la Resolución SBS 8514-2012, modificado por Resolución SBS 2150-2013, fijó como plazo para finalizar el cobro de la comisión por flujo el devengue de aportes obligatorios de enero 2023. Transitoriamente se ha establecido una comisión mixta que cobra la AFP por el servicio de administración, integrada por 2 componentes: una comisión porcentual calculada sobre la remuneración asegurable del afiliado (comisión sobre el flujo) más una comisión sobre el saldo del Fondo de Pensiones (comisión sobre el saldo).

7 El Marco Contable Conceptual del Manual de Contabilidad para las Administradoras Privadas de Fondos de Pensiones, vigente hasta el 31 de diciembre de 2012, señala que el reconocimiento de los ingresos por comisiones sobre aportes obligatorios, de acuerdo con lo establecido en la Ley del SPP y disposiciones de la Superintendencia de Banca, Seguros y AFP, debe efectuarse una vez acreditado en las respectivas Cuentas Individuales de Capitalización, el aporte obligatorio cotizado por los afiliados, hecho que conlleva a que las AFP realicen el proceso de conciliación previo que les permita identificar los aportes obligatorios efectuados para luego proceder con el proceso de acreditación respectivo.
} 
Es decir, bajo las anteriores normas contables de la SBS los ingresos por comisiones se reconocían como ingresos en base a lo percibido y no en base a lo devengado, es por ello que el Catálogo de Cuentas denomina al rubro Comisiones Recibidas que comprende el monto total percibido por la AFP por concepto de comisiones por la administración de los Fondos de Pensiones y por ingresos producto de regímenes de fraccionamiento de deudas previsionales. En consecuencia, el tratamiento contable de las comisiones no cumple con la NIC 18 al reconocer el total de las comisiones como ingresos en un mismo período, a pesar que el servicio por la administración de los aportes mensuales retenidos a los afiliados se dará hasta que éstos se jubilen.

\section{EFECTO DE LA NO APLICACIÓN DE LA NIC 18}

El efecto de reconocer anticipadamente los ingresos por comisiones (en base a lo percibido) en el período en que se cobran y, de otro lado, reconocer los gastos relacionados al servicio de administración de los fondos de pensiones en base a lo devengado, mejora los resultados de operación de las AFP y permite fortalecerlas patrimonialmente, e incluso habría permitido un mayor pago de dividendos a los accionistas de las AFP (distribución de utilidades).

Esta discusión no es nueva, por ejemplo en el 2002 el profesor Roberto Urrunaga señalaba que $e l$ sistema de comisión sobre remuneraciones ha sido conveniente para las AFP porque les ha permitido recuperar de manera más rápida y segura su inversión inicial (citado por Sergio Villegas en diario La Razón, viernes 12.07.2002 p.15).

\section{CAMBIO NORMATIVO E IMPLEMENTACIÓN GRADUAL DE LA NIC 18}

La Ley de Reforma del SPP modificó el artículo 30 del Texto Único Ordenado (TUO) de la Ley del Sistema Privado de Administración de Fondos de
Pensiones ${ }^{8}$ señalando que cuando las AFP cobren la comisión por retribución sobre la remuneración asegurable desde el día siguiente de la publicación de la Ley de Reforma del Sistema Privado de Pensiones, deberán realizar una provisión correspondiente a la retribución por la administración de los nuevos aportes, de acuerdo a la Norma Internacional de Contabilidad (NIC) 18 "Ingresos de Actividades Ordinarias".

El Anexo 1 de la Resolución SBS N 8513-2012 publicada el 08 de noviembre de 2012, modifica el Marco Contable Conceptual del Manual de Contabilidad para las Administradoras Privadas de Fondos de Pensiones, referido al "Reconocimiento del Ingreso por Comisiones sobre Aportes Obligatorios" señalando que el reconocimiento de los ingresos por comisiones por retribución sobre la remuneración (comisión por flujo) cobradas a los afiliados se reconocerán como ingresos devengados durante el período de permanencia del afiliado en la AFP, reconociéndose una vez acreditado el aporte obligatorio cotizado por los afiliados en las respectivas cuentas individuales de capitalización.

¿En qué consiste este cambio normativo?

Que a partir del 20 de julio de 2012, día siguiente de la publicación Ley $\mathrm{N}^{\circ} 29903^{9}$, los ingresos por comisiones por flujo cobradas se reconocerán como ingresos devengados durante el período de permanencia del afiliado en la AFP. Como la modificación del Manual de Contabilidad entró en vigencia a partir de la información correspondiente al mes de febrero $2013^{10}$, la provisión correspondiente a la retribución por la administración de los nuevos aportes correspondiente al período 2012, a partir del 20.07.2012, la registra en el período 2013 con cargo a resultados acumulados. ¿Y qué pasa con los ingresos por comisiones por flujo cobradas antes del 20.07.2012? Aparentemente quedan como están, reconocidas como ingresos bajo las normas contables anteriores, como veremos a continuación solo se provisionaría como ingresos diferidos los correspondientes a los afiliados que la AFP estima dejarán de aportar.

8 Aprobado mediante Decreto Supremo $\mathrm{N}^{\circ} 054-97-\mathrm{EF}$ y sus normas modificatorias.

9 Ley publicada el 19.07.2012, que regula que las AFP desde el día siguiente de la publicación de la Ley de Reforma del Sistema Privado de Pensiones, deberán realizar una provisión correspondiente a la retribución por la administración de los nuevos aportes, de acuerdo a la Norma Internacional de Contabilidad (NIC) 18 "Ingresos de Actividades Ordinarias".

10 La Resolución SBS N 771-2013 publicada el 02.02.2013 modificó la fecha de entrada en vigencia del Manual de Contabilidad para las Administradoras Privadas de Fondos de Pensiones, modificado por Resolución SBS Nº 8513-2012. 
Adicionalmente, el Anexo 1 de la Resolución SBS $\mathrm{N}^{\circ}$ 8513-2012 señala que tratándose de los afiliados por los que la AFP no perciba ingresos por comisiones, pero continúe prestando servicios, la AFP deberá constituir una provisión por ingresos diferidos, realizando un débito a la cuenta de ingresos 50706 denominada "Ajuste por provisión" y acreditando la cuenta 20501 denominada "Provisiones por Ingresos diferidos". Para determinar la referida provisión la AFP deberá tomar en cuenta, entre otras variables que señala el Anexo 1, un horizonte de tiempo no menor a 20 años y el estimado de los afiliados aportantes (cotizantes) que pasarían a ser no aportantes en el referido horizonte de tiempo.

¿A qué se refiere esta modificación?

Que los afiliados que ya no aportan, es decir quienes se encuentran desempleados y que anteriormente aportaron al fondo de pensiones (afiliados activos que no cotizan), ya pagaron por el servicio de administración de los aportes efectuados a su cuenta individual de capitalización y por lo tanto la AFP está obligada a prestar el servicio hasta la fecha en que se jubilen, por lo que la modificación señala que la AFP debe constituir una provisión por ingresos diferidos, es decir extornar los ingresos que fueron reconocidos bajo las normas contables anteriores (criterio de lo percibido), así mismo debe estimar la provisión correspondiente a los afiliados actualmente aportantes que pasarán a ser no aportantes, e ir devengándolos a medida que la AFP va prestando el servicio, para ello debe tomar un horizonte mínimo de 20 años.

¿Y cómo se reconocen los ingresos por comisión por saldo y comisión mixta?

Para el caso de la comisión por saldo, así como el componente de la comisión mixta correspondiente a la comisión por saldo, se reconocerá como ingreso en la oportunidad que esta se cargue al fondo administrado. El componente de la comisión mixta correspondiente a la comisión por flujo, se reconocerá bajo lo percibido, es decir una vez acreditado el aporte obligatorio cotizado por los afiliados en las respectivas cuentas individuales de capitalización.

Entonces, tenemos una mixtura para la implementación gradual de la NIC 18, la comisión por flujo se reconocerá como ingresos devengados durante el período de permanencia del afiliado en la AFP (de acuerdo a la NIC 18), los ingresos por comisiones por flujo anteriores de los afiliados que no aportan ( $y$ del estimado de quienes dejarán de aportar) serán extornados estimando la provisión por ingresos diferidos y se irán devengando a medida que la AFP va prestando el servicio, para ello debe tomar un horizonte mínimo de 20 años. Como la comisión por flujo y la comisión mixta se eliminarán en un plazo de 10 años y sólo quedará la comisión por saldo, el reconocimiento del componente por flujo de la comisión mixta seguirá reconociéndose bajo el criterio de lo percibido, por lo que en ese momento se completará la implementación de la NIC 18.

\section{COMISIÓN POR SALDO ¿A QUIÉN BENEFICIA?}

Para tratar de responder esta pregunta primero veamos algunas diferencias entre la comisión por flujo y la comisión por saldo:

1. La comisión por flujo cobra un porcentaje sobre la remuneración asegurable reduciendo el ingreso presente (remuneración) del afiliado a cambio de un mayor ingreso futuro (fondo de pensión). Como la comisión por saldo cobra un porcentaje sobre el saldo del fondo administrado, el afiliado no ve reducir su remuneración. Sin embargo, se reduce su ingreso futuro (fondo de pensión) porque de allí se descuenta la comisión de la AFP.

2. La comisión por flujo implica que el afiliado ha pagado por el servicio de administración del aporte mensual a su fondo por períodos sucesivos (hasta que se jubile), mientras que con la comisión por saldo el afiliado ha pagado el servicio de administración correspondiente a un período. Si el afiliado se queda sin empleo ( $y$ deja de aportar a su fondo de pensiones), bajo la comisión por saldo seguirá pagando por el servicio de administración y se le descontará del saldo de su fondo de pensiones.

3. La comisión por flujo hace complejo determinar la rentabilidad neta del fondo de pensiones del afiliado ya que el valor cuota ${ }^{11}$ publicado no

11 El valor cuota se obtiene dividiendo el valor total del activo del fondo, deduciéndole previamente el pasivo exigible, dividido por el número de cuotas emitidas. El rendimiento del fondo de pensiones de un determinado período se obtiene dividiendo el valor cuota final por el valor cuota inicial. 
incorpora el efecto de la comisión, mientras que en la comisión por saldo el valor cuota incorpora el efecto de la comisión, y permitiría comparar la rentabilidad con otros instrumentos financieros (alternativas de inversión).

Desde la perspectiva del afiliado, la comisión que lo beneficiaría (asumiendo que las tasas cobradas por la comisión por flujo y la comisión por saldo son equivalentes) dependerá de su preferencia por un mayor o menor ingreso presente, $y$ de su expectativa de empleo hasta su jubilación.

Desde la perspectiva de las AFP, en el corto plazo la comisión por flujo le permitirá contar con más disponibilidad de efectivo para invertir (en instrumentos permitidos por la regulación). Por otro lado, por normas contables las AFP han reconocido el total de la comisión como ingresos (criterio de lo percibido), lo que les ha permitido mejorar su situación financiera y patrimonial. Adicionalmente, les ha permitido mostrar una mayor rentabilidad de los fondos administrados al no incluir el efecto de la comisión cobrada a los afiliados.

Entonces, cuando se creó el SPP la única desventaja de la comisión por flujo era el efecto en la remuneración del afiliado (ingreso presente), razón por la que se dispuso un aumento de la remuneración de los trabajadores que se afiliaban al SPP, ¿casualidad?

\section{¿LA COMISIÓN POR SALDO ALINEA LOS INCENTIVOS ENTRE LAS AFP Y LOS AFILIADOS PARA BUSCAR MAYOR RENTABILIDAD?}

Bajo un esquema de comisión por saldo, el crecimiento del fondo estará en función de los aportes que efectué el afiliado y la rentabilidad neta del fondo (cuyos componentes son la rentabilidad de las inversiones menos la comisión cobrada por la AFP).

Si las inversiones efectuadas con los fondos administrados por las AFP obtienen un bajo rendimiento o pérdida, ¿̇la AFP seguirá cobrando la comisión por saldo?, la respuesta es afirmativa, hasta que el fondo (cuenta individual de capitalización) del afiliado tenga saldo, el que irá aumentando por los aportes mensuales.

Si el afiliado está desempleado (y por tanto, no aporta a su fondo), entonces su fondo crecerá en la medida que la rentabilidad de las inversiones sea mayor a la comisión por administración, caso contrario verá disminuir su fondo. En consecuencia, ¿en un escenario de recesión económica (y aumento del desempleo) las AFP verán una disminución sustancial en las comisiones cobradas a los afiliados bajo el esquema de comisión por saldo?

Para que realmente las AFP tengan incentivos en buscar mayor rentabilidad la comisión debe ser cobrada sobre la rentabilidad del fondo ${ }^{12}$ y no sobre el saldo del fondo. Esta propuesta tampoco es nueva, en el 2002 Mario Roggero, ex congresista del FREDEMO, señalaba que en la Comisión de Trabajo y Economía del Congreso se ha propuesto en el articulado del proyecto dos comisiones: una fija, que cubra los gastos administrativos de las AFP, y otra comisión basada en la rentabilidad de los fondos (citado por Sergio Villegas).

\section{CONCLUSIONES}

1. La Reforma del SPP dista de la propuesta de reforma planteada en el plan de gobierno durante la campaña electoral, y de la propuesta de los congresistas oficialistas Diez Canseco y Delgado.

2. No se ha permitido el ingreso de otros intermediarios financieros (bancos, cajas municipales, etc.) que hubieran generado una mayor competencia en el SPP, en especial para el caso del segmento de trabajadores independientes, para incorporarlos de manera gradual al SPP.

3. Sigue el cautiverio de los afiliados al SPP, no se han dado opciones de desafiliación que permitan al afiliado trasladar el fondo acumulado a una institución financiera que permita un retiro programado y obtener una mejor rentabilidad y menores costos de administración (comisión).

4. Las AFP han logrado que las principales propuestas del Plan de Gobierno y del proyecto de Ley 474/2011CR no se incluyan en la Ley de Reforma

12 Puede aplicarse sobre la rentabilidad real (descontando la inflación), establecer una tasa escalonada (tasa base y tasa de éxito en función a un benchmark por tipo de fondo), etc.

216/ QVIPVIRAMAYOC|Vol. 22(41) 2014 
del SPP; es decir, se desvirtuó que: el SPP sea complementario y no obligatorio; ingresen otras empresas del sistema financiero como administradores de otros productos financieros con fin previsional; la comisión que cobran por la administración de los fondos de pensiones se calcule sobre la rentabilidad del fondo; y la implantación inmediata de la NIC 18 . No les quedó alternativa que aceptar la aplicación de la NIC 18, aunque lograron que su implementación sea gradual y que por las comisiones cobradas a aquellos afiliados que se estima no queden desempleados hasta su jubilación, no se efectué la provisión por ingresos diferidos, lo que disminuye el efecto sobre el patrimonio de las AFP.

5. La aplicación de la comisión por saldo asegura de alguna forma a las AFP el cobro de comisiones de aquellos afiliados que quedan desempleados ante escenarios de recesión económica, y con la incorporación obligatoria de los independientes al SPP o al sistema público, verán aumentar el cobro de comisiones. Adicionalmente, las políticas públicas de disminución de la informalidad y no aumento de la Remuneración Mínima Vital $(\mathrm{RMV})^{13}$ permitirían que más trabajadores se incorporen al SPP. ¿A quién cree Ud. que más benefició la Reforma del SPP?, definitivamente no a los afiliados.

\section{REFERENCIAS BIBLIOGRÁFICAS}

1. Consejo Normativo de Contabilidad (2012). Norma Internacional de Contabilidad 18 Ingresos de Actividades Ordinarias. Portal electrónico: http://www.mef.gob.pe/contenidos/conta $\mathrm{publ} / \mathrm{con}$ nor_co/vigentes/nic/NIC_18_ BV2012.pdf

2. Diez Canseco, J.y Delgado, J. (2011). Proyecto de Ley 474/2011CRLey de fortalecimiento y transparencia del sistema privado de pensiones para beneficio de los usuarios y afiliados. Congreso de la República, Lima, Perú.

3. El Comercio (2011). Portal electrónico: http:// elcomercio.pe/politica/gobierno/humala-dice-que-no-tocara-afp-su-plan-gobierno-siembra-dudas-noticia-745116

4. Gana Perú (2010). Portal electrónico. Plan de Gobierno 2011-2016, diciembre 2010. http:// www.presidencia.gob.pe/plan-de-gobierno-gana-peru-2011-2016

5. Lineamientos Centrales de Política Económica y Social para un Gobierno de Concertación Nacional. http://www.presidencia.gob.pe/images/ archivos/ollanta_humala_hoja_de_ruta.pdf

6. Instituto Peruano de Economía (2010, 2011, 2012). Portal electrónico, diversas fechas. http:// www.ipe.org.pe/comentario-diario

7. Ley N ${ }^{\circ} 29903$ Ley de Reforma del Sistema Privado de Pensiones publicado el 19 de julio de 2012 en diario El Peruano.

8. Resolución SBS N ${ }^{\circ} 8513-2012$ publicada el 08 de noviembre de 2012 en diario El Peruano.

9. Villegas, S. (2002). Expertos hablan sobre las AFP. Diario La Razón 12.07.2002, p. 15, Lima, Perú.

13 Según el Instituto Peruano de Economía (IPE) una RMV baja tendría poco efecto sobre el empleo, pero una elevada (RMV) lo reduciría significativamente (Comentario Diario del 03.09.2010). 